\title{
“Cerchiamo un bambino distinto”. La Genesi di BeLLisSima NEI SOGGETTI DI CESARE ZAVATTINI
}

\section{CRISTINA JANDELLI}

\begin{abstract}
Summary: This contribution investigates, from a philological-historical perspective, the different versions of texts by Cesare Zavattini that will later merge into the subject of "Bellissima", the film directed in 1951 by Luchino Visconti, starring Anna Magnani. Through a crucial decade for Italian cinema, the early forties and fifties of the twentieth century, the variants depict mutable scenarios through deep historical, political and cultural changes. From Fascist Cinecittà, animated by its stars swept away by the Second World War, to the change of gender of the two protagonists-first a widower and his son, then a woman willing to barter her daughter in exchange for the realization of their dreams- the variants of the subject draw a long-standing perspective on modern society: life becomes a show.
\end{abstract}

Deluso dal mondo del cinema che ha scartato al provino suo figlio, il cameriere protagonista di una deludente avventura a Cinecittà sbatte la porta in faccia alla compagnia di guitti teatrali che l'aveva illuso. Così Cesare Zavattini chiude il primo soggetto della lunga scia di trame che condurrà fino a Bellissima di Luchino Visconti.

Il film del 1951, tratto da un soggetto di Zavattini ma sceneggiato da Suso Cecchi D’Amico, Francesco Rosi e dallo stesso Visconti, anticipa alcune forme e temi della modernità cinematografica ${ }^{1}$. La riflessione metalinguistica, anzitutto: i personaggi si muovono all'interno dell'apparato produttivo nazionale che fa da sfondo alla vicenda; la macchina da presa entra nei luoghi segreti di Cinecittà, ne ritrae i giardini, la piscina all'aperto, i teatri di posa, le cabine di montaggio, le salette di proiezione. A livello tematico il film anticipa l'affermazione dei miti dello spettacolo di massa: il mondo del cinema all'apparenza promette alle classi più umili una rapida scalata sociale grazie alla concezione illusoria dello spettacolo inteso come luogo dell'evasione e del riscatto. Anna Magnani/Maddalena sogna ad occhi aperti nell'arena estiva gremita dove si proietta Il fiume rosso di

1 "Cesare Zavattini, come si sa, non partecipò, se non informalmente e occasionalmente al successivo lavoro di trattamento e sceneggiatura; ma è innegabile il grosso debito, non solo a livello del plot e della story, che ha Bellissima nei suoi confronti”. (Lino Micciché 200) 
Howard Hawks (1948): "A Spartaco, nun me capisci tu. Guarda che bei posti... guarda noi 'ndo vivemo. Io quando vedo 'ste cose qua, mah ... 'n so' favole, 'n so' favole". La protagonista di Bellissima, Maddalena Cecconi, è una donna che crede vere le favole e vede nella figlia - sgraziata, bruttina, priva di ogni appeal - un mezzo per la propria autoaffermazione. Cinecittà alla fine le rivela il suo volto beffardo e l'unica reazione possibile prima di perdersi davanti all'esplicito tentativo di corruzione morale (due milioni di lire per il contratto di scrittura della figlia) sarà la marcia indietro: la "fanatica" Maddalena intima al marito Spartaco di buttar fuori gli emissari della produzione dal caseggiato vociante della periferia romana che Visconti ha precedentemente ritratto dal basso come una minacciosa prigione. Di lì madre e figlia non saranno più capaci, metaforicamente, di uscire. La bambina disegnata da Visconti è una figura senza qualità, una balbuziente introversa, sorta di burattino nelle mani della madre, sottomessa alla sua volontà ("Tu devi dare retta solo a mamma”) e schiava inconsapevole dei suoi sogni: l'unico sentimento che ne muove le azioni è quello di compiacere la volitiva e vitalistica Maddalena. Maria, cioè, appare molto diversa dai bambini che popolano i film di De Sica e Zavattini, dal Pricò de I bambini ci guardano (1942) alla banda di strada degli Sciuscià (1946) fino al Bruno di Ladri di biciclette (1948). Questi maschietti impertinenti riescono in varie circostanze a ritagliarsi una loro libertà d'azione rispetto al mondo dei grandi che intende manovrarli e manipolarli: perfino Pricò, il più sottomesso del gruppo, nel finale volta le spalle alla madre avviandosi vestito di nero in campo lungo verso la sua vita da orfano. Analogamente Bruno prende per mano suo padre nel finale del film e con questo gesto adulto lo consola, consapevole della vergogna che l'uomo prova - per non dire dei bambini di Sciuscià che si contrappongono frontalmente al mondo degli adulti turlupinandoli. Maria no. La bambina con le trecce lunghissime tagliate per esigenze di copione che, durante il fatidico provino, si mette a piangere con la bocca spalancata come un personaggio dei fumetti, non gode di alcuna libertà di manovra. Il suo destino somiglia a quello delle donne degli anni cinquanta appena iniziati, domate e dominate dalla nascente società dei consumi: è segnato, come quello di Maddalena, dalla pressante richiesta sociale di un convenzionale rientro dentro le mura domestiche. Per la seconda volta, dopo la prima guerra mondiale e il fascismo, alla donna italiana del novecento è intimato il ritorno all'ordine morale cattolico borghese ${ }^{2}$. Non che la sinistra delinei un'alternativa: sia Zavattini che Visconti corroborano l'assunto ideologico

2 Cfr. Lucia Cardone 204-7. 
garantendo il ritratto di una indimenticabile figura materna, quella di Maddalena Cecconi, tanto desiderosa quanto incapace di affrancarsi dal proprio ruolo sociale cedendo alla seduzione del denaro e del successo ("Io pure, se avessi voluto...").

Con il soggetto di Bellissima e con il cambio di sesso, il personaggio del bambino co-protagonista del racconto insieme all'adulto genitore, sorta di costante poetica dell'universo narrativo zavattiniano, subisce dunque un'evidente trasformazione rispetto alle figure delineate nei film prebellici e neorealisti realizzati da De Sica in stretta collaborazione con il suo sceneggiatore d'elezione. Tentare di spiegare i motivi di questa trasformazione e seguirla nei suoi sviluppi implica un ritorno ai documenti che può permettere di colmare qualche lacuna storica e interpretativa.

Sfogliando il fascicolo Bellissima che contiene dattiloscritti e manoscritti autografi di Cesare Zavattini, depositato presso la Biblioteca Panizzi di Reggio Emilia, si scoprono otto testi battuti a macchina, alcuni contenenti note vergate a mano più una scaletta divisa in punti. La datazione dei documenti resta incerta perché nessuno dei fogli reca in calce o in testa la data: ma ci sono fondati motivi per ritenere che il soggetto iniziale, schedato come R9a (Cerchiamo un bambino distinto) sia precedente o coevo al 1942. In ogni caso l'idea di un padre che accompagna suo figlio a Cinecittà per partecipare a un provino, illuso da una compagnia di guitti sulla possibilità di lanciare il ragazzino nel mondo del cinema, precede la stesura del soggetto La bambina più bella del mondo dove, negli stessi ruoli, compaiono una madre e una figlia ${ }^{3}$. Già il fatto in sé che non sia un uomo a sognare di far entrare nel mondo del cinema suo figlio, ma una donna sua figlia, appare significativo: infatti nella tradizione italiana (e non solo) le seduzioni della celluloide si sposano con le accuse mosse contro il genere femminile fin dalle origini del cinema negli scritti che stigmatizzano la nuova forma di spettacolo. Prima le fiere, poi le sale stabili costituiscono altrettante lusinghe per giovani madri a caccia di sogni segreti in compagnia dei figli: duplice rapimento, duplice annientamento nelle malìe diseducative della finzione. In quanto intrinsecamente fragili, donne e bambini appaiono come le vittime designate della perdizione a mezzo cinema ${ }^{4}$.

3 Pio Baldelli (102), nella sua monografia su Visconti, scrive che "il primo soggetto", schedato nel fondo come R9f, "venne steso da Zavattini in due paginette (riprendendo uno spunto, quel La bambina più bella del mondo, che è addirittura del 1942)." Dal momento che i soggetti schedati con il titolo Cerchiamo un bambino distinto (R9a, R9b) sono precedenti ai due de La bambina più bella del mondo (R9c, R9d), bisogna ritenerli coevi o anteriori al 1942.

${ }^{4}$ A questo proposito si vedano Silvio Alovisio, (269-286) e Luca Mazzei, (257-268). 
Complesse e stratificate orditure testuali costituiscono la norma nella lunga avventura cinematografica dello scrittore luzzarese, considerando fra l'altro che i film realizzati dai suoi soggetti sono di gran lunga minori rispetto ai progetti che non hanno mai visto la luce. Zavattini vive sommerso di carte, apre e chiude fascicoli incessantemente, riadatta e riscrive vecchi soggetti attualizzandoli. Passeggia per il suo piccolo studio, detta alla segretaria, poi cancella e modifica ancora, spesso nottetempo, assediato dall'insonnia, quando non dipinge. Le sue fabule sono tutte molto simili nella loro laconica brevità di paginette dattiloscritte con ampi margini, somigliano a orditure di intrecci minimi suscettibili di infinite variazioni e modifiche, spesso attraversano i decenni, in particolare quello centrale del novecento che prende il via ai margini dell'entrata in guerra e si conclude in piena fase di ricostruzione. Un mondo è tramontato e un altro sta sorgendo: nel bel mezzo il cinema italiano, con il neorealismo, ha dato vita a una nuova concezione del film che fa perno anche su quei soggettini, su quei foglietti di carta leggera continuamente riscritti nel corso del tempo 5 . I nove documenti contenuti nel fascicolo Bellissima vanno considerati varianti successive di una catena intertestuale lunga quasi un decennio: l'ambientazione dell'avventura a Cinecittà da una parte e la coppia protagonista padre/figlio (poi madre/figlia) dall'altra appaiono i capisaldi dell'intreccio metacinematografico. Tutto il resto cambierà ruotandovi attorno e il film di Visconti, non sceneggiato, come si è detto, da Zavattini, diventerà l'anello che per la verità non salda la cesura fra un'idea di un racconto e la sua traduzione in film: a livello storico-critico Bellissima si è letto come un tassello fondamentale dell'opera viscontiana, per di più segnato da una straordinaria prova d'attrice di Anna Magnani, mentre lo si è ricondotto in modo assai più generico all'interno della poetica zavattiniana ${ }^{6}$.

Il protagonista di Cerchiamo un bambino distinto è un cameriere di spirito, vivace e arguto, uno che tratta alla pari anche gli avventori più ragguardevoli: ha le movenze di un comico, anche se la storia lo condurrà verso esiti patetici. Conoscendo la passione zavattiniana per le corrispondenze onomastiche, il nome Fabrizio induce a pensare che il personaggio sia stato scritto pensando ad Aldo Fabrizi. Fino dal 1940, del resto, lo scrittore aveva caldeggiato il passaggio dei comici del varietà al cinema e Fabrizi era uno degli interpreti preferiti dallo scrittore che, sbarcato dalla provincia emiliana nella

\footnotetext{
${ }^{5}$ Cfr. Millicent Marcus, Italian Film in the Light of Neorealism.

6 "Cesare Zavattini, come si sa, non partecipò, se non informalmente e occasionalmente al successivo lavoro di trattamento e sceneggiatura; ma è innegabile il grosso debito, non solo a livello del plot e della story, che ha Bellissima nei suoi confronti". (Micciché 200)
} 
capitale si intratteneva volentieri con il varietà7. Non a caso Fabrizi debutterà due anni più tardi in un film sceneggiato da Zavattini, Avanti c’e posto di Mario Bonnard ${ }^{8}$. Negli anni che precedettero il passaggio cruciale dell'attore romano dallo spettacolo di varietà al decisivo ruolo drammatico di Roma città aperta (Roberto Rossellini, 1945), lo scrittore lo aveva già idealmente eletto quale volto decisivo del nuovo cinema italiano.

Tornando al soggetto, quando Fabrizio fa rientro a casa nel condominio popolare dove abita, trova ad aspettarlo suo figlio Enrico. L'uomo è vedovo e ripone nel bambino di sei anni grandi speranze. Perciò risponde subito all'annuncio della casa di produzione Fulgor che cerca "un bambino dai sei ai nove anni dall'aspetto distinto per interpretare un film" ${ }^{9}$. Non tanto paradossalmente, Zavattini negli anni successivi parteciperà attivamente alle ricerche di casting per realizzare i capolavori di De Sica, protagonisti bambini della stessa età di Enrico, anche se l'aspetto distinto sarà l'ultima delle preoccupazioni dell'attore-regista, come si evince da questa testimonianza relativa alla lavorazione di Ladri di biciclette (1948):

Gli interpreti li trovammo in modo avventuroso. Il grande problema fu il bambino. Me ne portarono centinaia: o erano bellini, romantici, lisciati, o erano incapaci. I bambini sono attori per eccellenza: hanno espressione, innocenza, spirito, autenticità, pudore .... Ricevevo i bambini candidati ai miei film: si presentavano in lacrime, più di sorpresa che di paura .... A un tratto nella fila dei genitori, vidi un operaio che teneva il figlioletto per mano .... Era Lamberto Maggiorani ..... Intanto il bambino non si trovava. Disperato, decisi di cominciare ugualmente il film. Iniziai con la scena di Maggiorani che va in cerca dell'amico che lo aiuti a ritrovare la bicicletta. Si girava in quella specie di teatrino da dopolavoro. Stavo dicendo qualcosa a Maggiorani, quando mi volto infastidito dai curiosi che mi si affollavano intorno e vedo uno strano bambino con

7 Lattività di Zavattini recensore del teatro di varietà sul settimanale "Tempo" si svolge dal 1940 al 1941. Nella rubrica dedicata al comico romano, scrive: "La sua faccia stessa, cosi cinema, mobile - quando canta Lulù non sei più tu — subisce la sua immaginazione: gli occhi hanno il morbo di Basedoff e la mascella diventa un primo piano crudo e enorme. Di tutti i comici italiani mi sembra il meno leggiadro e il più sarcastico, un osservatore con i nervi un po' scossi." Cesare Zavattini, Aldo Fabrizi).

8 In Avanti c’è posto Fabrizi riproponeva il personaggio del tranviere che lo aveva reso famoso nel varietà: Zavattini collaborò con lui e con Fellini, che scriveva i monologhi teatrali dell'attore, al soggetto del film, poi ne firmò con altri la sceneggiatura.

9 R9a, p. 3. Nella seconda versione, contrassegnata come R9b, Zavattini apporta solo qualche ritocco a livello formale. 
una faccia tonda, e un nasone buffo, e stupendi occhi vivissimi. Enzo Staiola. Questo me l'ha mandato San Gennaro, pensai ${ }^{10}$.

Dal momento in cui il cameriere risponde all'annuncio della casa di produzione, iniziano le peripezie erratiche di padre e figlio, schema che si riproporrà identico proprio in Ladri di biciclette. Ma la città di Cerchiamo un bambino distinto è diversa da quella che fa da sfondo all'avventura dei due indimenticabili personaggi del cinema neorealista: è il centro di produzione dello spettacolo di massa, un microcosmo dove si muovono divi e comparse, guitti e direttori di produzione, giovani prime attrici e generici nel contesto di teatrini di periferia dove si svolgono le prove, locali affollati per le audizioni e piccole salette di proiezione. Nella Cinecittà raccontata dal soggetto del 1942 "ogni tanto passa qualche divo, Nazzari o De Sica, Isa Miranda o Clara Calamai o Doris Duranti, e tutti si precipitano loro intorno per presentare i figli e chiedere autografi": l'adulto e il bambino finiscono per perdersi in quel labirinto che è il più grande stabilimento cinematografico italiano sorto sulle ceneri della vecchia casa di produzione Cines e inaugurato dal Duce, cioè il luogo dove si girano i film dei telefoni bianchi e i drammi storici di cui gli attori Vittorio De Sica e Amedeo Nazzari sono gli esponenti di spicco: il primo viene diretto da Mario Camerini in successi popolari come Gli uomini che mascalzoni (1932) e Il signor Max (1937), il secondo è noto come prestante eroe in costume protagonista di Ginevra degli Almieri di Alessandrini (1935) e de La cena delle beffe di Blasetti (1941). Mentre i riferimenti a Isa Miranda, Clara Calamai e Doris Duranti verranno espunti dalle successive varianti del soggetto, Nazzari e De Sica resteranno menzionati, vedremo in seguito perché.

Dopo aver reagito all'incapacità del figlio a recitare tirandogli il primo schiaffo della sua vita - gesto prevaricatore che compiranno sia Antonio Ricci con Bruno in Ladri di biciclette che Maddalena con la piccola Maria in Bellissima, ma soprattutto l'io narrante de La notte che ho dato uno schiaffo a Mussolini, zibaldone scritto da Zavattini nel 1976 - Fabrizio "se ne va col figlio a una rappresentazione di un piccolo teatro di legno della periferia. Si tratta di una compagnia di guitti che agli occhi di Fabrizio sembrano dei grandi attori" 11 . Giunta all'apice, la frenesia di rivalsa socia-

10 Le dichiarazioni sono riportate in Paolo Nuzzi, Ottavio Iemma,102-3. Quanto a Zavattini, anche a lui capitava di mettere la propria esperienza al servizio della ricerca degli interpreti: "Ci sono state scelte di attori alle quali ho partecipato anch'io. Per esempio, quella del bambino di Ladri di biciclette, di cui avevo visto i provini insieme a quelli di tanti altri bambini" (Paolo Nuzzi, Ottavio Iemma 102-3).

11 R9a,m p. 6. 
le dell'uomo si ripercuote sul figlio che "sta lì con i suoi grandi occhi timidi, senza essere più capace di riconoscere il padre, diventato quasi timoroso di lui" 12 .

Nel film di Visconti è la ridicola insegnante di recitazione Tilde Spernanzoni a istruire Maria utilizzando cascami inservibili del sistema Stanislavskij che il regista mette alla berlina ${ }^{13}$; nel soggetto di Zavattini il ruolo è tenuto dal direttore di una compagnia teatrale di terz'ordine, di quelle che ancora battevano la provincia e le periferie nell'Italia pre-bellica, il "tipo classico di fallito presuntuoso e scroccone" (R9a 7). Di qui alla fine dell'avventura a Cinecittà, Fabrizio verrà tallonato dai guitti che hanno a cuore solo i pasti e i vantaggi materiali che possono venire dall'istruire il piccolo Enrico. Fabrizio stesso agisce per calcolo, permette ai guitti di attorniarlo perché "il lavoro di cui Enrichetto dovrebbe essere il protagonista, qualora sia prescelto, è un noto lavoro di cui esiste anche il dramma per le scene teatrali" (R9c 8). In questa descrizione si può leggere in filigrana la conoscenza di una pratica spettacolare molto diffusa nel cinema e nel teatro italiani del Ventennio dove gli attori riproponevano lo stesso personaggio incarnato al cinema anche nei teatri dei piccoli centri cittadini sfruttando intensivamente la popolarità del cinema.

Enrichetto, una volta approdato davanti alla macchina da presa, si comporta esattamente come Maria nel film di Visconti: fa una prova disastrosa. Ma la causa non è l'improvvisa crisi di pianto: "il peggiore è Enrichetto per il suo modo lezioso di recitare, proprio come glielo hanno insegnato quei volgari guitti che infestano la sua casa. Quella recitazione indigna o fa ridere" (R9c 9), si legge nel soggetto. Dietro quei guitti e i loro insegnamenti c'è un mondo che all'inizio degli anni Cinquanta non poteva più essere rappresentato perché veniva percepito come appartenente a un passato già lontanissimo, quello dell'introduzione del sonoro: verso la metà degli anni Trenta, infatti, le riviste teatrali Comoedia e Scenario avevano intrapreso una campagna per l'affermazione, in Italia, di una recitazione cinematografica naturalistica lontana dalla tradizione teatrale. Zavattini amava gli attori del varietà e del teatro popolare per la stessa ragione: mancando loro una tradizione illustre potevano muoversi in libertà, intrattenere il pubblico astenendosi dal "birignao" imperante anche nel cinema dell'epoca. Saranno gli attori del varietà Fabrizi e Magnani, difatti, i protagonisti della nuova stagione

12 Analogamente, nel soggetto La bambina più bella del mondo, con protagonista Mariù, lei "sta lì con i suoi grandi occhi ingenui, senza essere più capace di riconoscere il padre, diventata quasi timorosa di lui". R9c 8.

13 Cfr. Siro Ferrone 88. 
cinematografica nel dopoguerra ${ }^{14}$.

Il mondo ridicolo degli attori guitti e "cani" viene precisato in una nuova versione del soggetto cui è allegata una paginetta recante il titolo "Soluzione relativa alla protagonista donna". Si tratta di una variante che mette al centro dell'azione di Fabrizio ed Enrichetto la loro domestica, Anna (Magnani?15), "un carattere pieno di immaginazioni”, segretamente amata dal vedovo e circuita dal direttore della compagnia che in questa variante assume i tratti che in Bellissima saranno dell'Annovazzi di Walter Chiari: "Anna prende parte alle recite, è sempre stato un sogno anche per lei recitare, e il direttore solletica in lei questa passione e le lascia credere di aver anche lei delle doti non comuni. Forse Anna seguirà il direttore nella sua vita di comico vagante" 16 . Tale descrizione già si confa al personaggio delineato da Anna Magnani ma ricorda anche quello interpretato da Carla Del Poggio in Luci del varietà di Fellini-Lattuada (1951) che mette in scena lo stesso mondo di attori girovaghi evocato dai soggetti zavattiniani. Alla fine però la ragazza apre gli occhi e capisce "chi è il direttore, in quale grottesco e basso mondo stava per entrare" 17 . In entrambe le varianti, con protagonista maschile o femminile, trapela dunque un duro giudizio morale contro i "comici vaganti", siglata dal gesto finale di Fabrizio che chiude dignitosamente la porta in faccia ai guitti scrocconi. Anche Maddalena in Bellissima farà cacciar fuori di casa i rappresentanti del mondo fasullo del cinema. L'accusa per Zavattini resta sempre la stessa: lo spettacolo guasta, disgusta e corrompe l'innocenza dei bambini.

Il soggetto successivo, dal titolo La bambina più bella del mondo, riparte dalla versione precedente del testo integrata con la trama di Anna ${ }^{18}$. Il primo dattiloscritto è cioè una copia di Cerchiamo un bambino distinto cui Zavattini ha apportato numerose correzioni a mano. Intanto cambia il

14 "Una recitazione, una messinscena più naturale la chiedono tutti: pochi anni dopo saremo anche ufficialmente in piena battaglia contro la commediola sentimentale, intimista." (Sandro Bernardi 154).

15 Zavattini ammira anche Anna Magnani prima sul palcoscenico del teatro di rivista che sullo schermo, e puntualmente ne annota la bravura. L'attrice, giovanissima, è inoltre, con Riento, nel cast di Teresa Venerdì (1941), debutto "segreto" della coppia Zavattini-De Sica.

16 La pagina, non numerata, si trova in coda ai documenti R9a 11 e R9b 11.

17 Ibidem.

18 Non sono però le "due paginette" di cui parla Baldelli che riproduce, come già notato, R9f. Sul documento, di dieci pagine, contrassegnato come R9c, Zavattini ha apportato numerose correzioni a mano. 
nome del protagonista: diventa Riento, cognome del noto attore di varietà Virgilio passato dalla fine degli anni Trenta al cinema e già nel cast di Teresa Venerdì di De Sica ${ }^{19}$, probabilmente indicato a questa altezza cronologica come possibile protagonista del film. Nel ruolo principale il soggettista mantiene un caratterista comico, ma gli affianca stabilmente una protagonista femminile, ancora di nome Anna, mentre il posto del bambino, Enrico, è preso da una bambina, Mariù ${ }^{20}$. "Anna ... pettina Mariù in mille modi, le prepara i vestiti, recita con lei", prefigurazione delle sequenze girate da Visconti dove Maddalena pettina Maria con i capelli "tutti indietro, come sta mamma". Un'altra variante riguarda proprio la bambina, che per la verità "reciterebbe benissimo, con grazia e vivacità: ma la concezione del teatro che ha il nostro Riento è terribilmente filodrammatica sicché per lui Mariù non recita secondo le regole" (R9d 7). Ne La bambina più bella del mondo il suo apprendistato è affidato al "primo attore giovane" della compagnia di guitti, "Avanzi, un tipo classico di fallito presuntuoso e scroccone sulla trentina" (R9d 8). Come nell'altro soggetto, la parte centrale dell'intreccio è costituita dalle prove della commedia di cui il film costituirà l'adattamento cinematografico: l'appartamento si trasforma in un palcoscenico e durante le prove Avanzi corteggia Anna ma solo per impadronirsi dei suoi miseri risparmi. I tratti essenziali di Avanzi sono gli stessi di Annovazzi: anche se il personaggio del film verrà variato da Visconti, il seduttore maneggione di Walter Chiari corteggia analogamente la protagonista per interesse.

Nel panorama del teatro italiano di fine anni Trenta, i termini "guitto" e "filodrammatico" non erano sinonimi: con il primo si designavano attori professionisti, ma di terz'ordine, che si spostavano lungo la penisola su carretti e furgoni come i comici dell'avanspettacolo di Fellini e Lattuada; con il

19 Nella già citata rubrica dedicata al varietà, Zavattini si dichiara antico estimatore del comico: "Gli abbiamo visto fare — scrive — il maestro d'orchestra con la solita mimica straordinaria, tirava su gli applausi dalla platea come ostriche, il suo guardare dall'alto in basso con gli occhi languidi troncava ogni ritegno." Cesare Zavattini, Inaugurazione del Reale.

20 Il nome Mariù fa pensare a Mariù Pascoli, la bambina trovata grazie a un provino a Cinecittà per interpretare Ombretta, la protagonista di Piccolo mondo antico di Mario Soldati (1942). Cfr. Vittorio Calvino ["Si è trovata 'Ombretta.' Mariù diventa diva” Film Vol. 3, Settembre 21: (1940)] e l'articolo di Patrizia Bettella in questo numero di Quaderni d'italianistica. Ringrazio l'autrice per la segnalazione. A sua volta il nome dato alla bambina rimanda all'enorme successo popolare ottenuto dalla canzone Parlami d'amore Mariu interpretata da Vittorio De Sica nel 1932 in Gli uomini, che mascalzoni di Mario Camerini. 
secondo dilettanti che riproponevano sui palcoscenici cittadini i peggiori vizi della recitazione declamatoria dell'Ottocento, aggravati dalla scarsa dimestichezza con le scene e dal recupero infaticabile di vecchi repertori. Guitti e filodrammatici - questi ultimi inseriti anche nella peripezia di Antonio Ricci in Ladri di biciclette, nelle sequenze dello scantinato - rappresentano, in modo opposto e complementare, le peggiori eredità della recitazione teatrale italiana. Questi cascami di uno stile ormai divenuto cliché si infrangono contro lo stile mimetico e naturalistico di cui, negli anni che precedettero la stagione neorealista, si invocava l'avvento anche sulle pagine della rivista Cinema, che aveva visto Zavattini fra i collaboratori più autorevoli21. Il sottotesto del soggetto pone in evidenza la concezione degli attori e della recitazione dello scrittore luzzarese, orgoglioso detrattore del teatro di prosa fin dalle stroncature giovanili delle messinscene pirandelliane: solo i comici, cioè gli attori monologanti del varietà, e gli interpreti non progettati, cioè i bambini capaci di conferire un'esuberante spontaneità alla recitazione, possono costituire le risorse cui il cinema deve attingere per rinnovarsi 22 . La tradizione teatrale va abbandonata e insieme ad essa è necessario rigettare uno dei cardini su cui poggiava il cinema italiano degli anni Trenta-Quaranta, cioè lo stile di recitazione, tipico del teatro all'antica italiano, impostato su voci stentoree, contrassegnato dalla dizione inappuntabile e costipato dal vizio dell'acuto nasale: tali cliché erano rifioriti nell'alveo del cinema grazie all'avvento del sonoro e al doppiaggio dei film americani.

Le versioni successive del soggetto, che porta ormai il titolo definitivo di Bellissima, sono le più note: rispettivamente due, tre e undici pagine in cui compaiono tutti i principali elementi narrativi che costituiscono l'intreccio del film di Visconti23. La seconda, intessuta di note manoscritte, esordisce così: "Maddalena ha una figlia di sei anni, Maria. Maria non è molto bella, ma per sua madre è bellissima, lo dice sempre. Maria ha anche un leggero difetto di pronuncia e Maddalena non lo trova un difetto ma

21 Sui cliché della recitazione cfr. Claudio Vicentini.

22 Cfr. Cristina Jandelli, La scena pensante. Cesare Zavattini fra teatro e cinema.

23 Si tratta rispettivamente dei documenti R9e, R9f, R9h, citati rispettivamente come S2, S1 e S3 in Lino Micciché, Visconti e il neorealismo 199-200. Secondo Micciché "come molte 'messe in scena' viscontiane, questa [di Bellissima] è (anche) la 'lettura critica' di un testo, il soggetto zavattiniano" (Micciché 201). Sembra dello stesso avviso Zavattini quando scrive, dopo la visione del film: "La storia di Bellissima è buona, si vede che è tale, i mutamenti viscontei non l'hanno strutturalmente cambiata." Cesare Zavattini, 2002171. 
una qualità" (R9e 1). Dunque già nel racconto zavattiniano Maria è una bambina non solo ordinaria, ma svantaggiata rispetto alle coetanee: "La povera Maria è davvero stanca, ha subito la mania materna perché lei è timida ma non ha proprio nessuna particolare qualità. Si è messa a piangere un paio di volte e la madre ha fatto quello che non ha mai fatto, le ha dato perfino uno schiaffo" (R9e, p. 2. Il corsivo è mio).

Lo sfondo teatrale si è dissolto, il mondo del cinema in quanto fabbrica delle perfette illusioni incombe su madre e figlia: Maria viene mandata a lezione presso la scuola di danza della Ruskaia (parodia della moda persistente dei balletti russi) e c'è un'attrice, che a Cinecittà fa la comparsa, chiamata da Maddalena per togliere a Maria il suo vizio di pronuncia (nel soggetto di tre pagine Maddalena porta Maria all'Istituto di Rieducazione della Voce a Montesacro). Della compagnia dei guitti non è rimasta traccia. Per la prima volta, nel soggetto di undici pagine, Zavattini introduce un riferimento metacinematografico e autobiografico a Prima comunione di Alessandro Blasetti (1951, soggetto e sceneggiatura sue, protagonista Aldo Fabrizi) che va considerato il motivo occulto della presenza del "regista con gli stivali" nel ruolo di se stesso nel film di Visconti (ma nella sceneggiatura definitiva scomparirà ogni rimando al film, lasciando sospeso il gioco zavattiniano di mise en âbime) ${ }^{24}$. In questo nuovo soggetto, a riprova del fatto che il rimaneggiamento avviene verso l'inizio degli anni Cinquanta, al posto delle dive dei telefoni bianchi, di Amedeo Nazzari e di Vittorio De Sica, ci sono Ingrid Bergman e Roberto Rossellini che attraversano Cinecittà sulla loro automobile, mentre Maddalena si lancia a caccia di un autografo di Silvana Mangano, reduce dal successo popolare di Riso amaro di De Santis (1949)25. La citazione del film di Blasetti, in lavorazione nel 1950, permette di datare il documento come appena anteriore alla vendita del soggetto e della sceneggiatura di Bellissima da parte di

24 "Blasetti ha attraversato la regia del cinema italiano per decenni e il regista che fa il Regista, anzi il regista che fa se stesso, diviene qui momento di consapevolezza sociale del mestiere e della gente di cinema; ma anche prova rispettosa dei sentimenti e delle illusioni involontariamente indotte - nella madre Anna Magnani, una "regista" cialtrona della propria bambina, ovvero della proiezione in lei della fatica e della crudele casualità del successo - dai registi-incantatori nella nostrana e casereccia fabbrica dei sogni del dopoguerra, prima del boom economico." (Fabrizio Borin 281).

25 "Siccome Maddalena desidera tanto un autografo di Silvana Mangano che sta girando un film al teatro n. 4, Annovazzi ce l'accompagna e riesce a ottenere facilmente dall'attrice l'autografo. Tutto questo incanta Maddalena e la convince che Annovazzi è davvero onnipotente. Per questo Maddalena nella penombra 
Zavattini26: il mondo dello spettacolo italiano appare profondamente modificato rispetto a quello pre-bellico presente nei soggetti degli anni quaranta, quelli all'origine della filiera. Dopo la temperie neorealista, i modi di produzione in Italia vengono riconfigurati secondo il modello hollywoodiano e rinasce il divismo ${ }^{27}$.

Là, nell'immenso teatro numero cinque, il regista ha finalmente cominciato i provini con un collaboratore eccezionale, Aldo Fabrizi, interprete del film. Tutte le candidate devono fare una scena del film con Fabrizi e Maddalena cerca di diventare amica con l'illustre attore colmandolo di elogi; gli fa perfino vento quando le sembra che Fabrizi abbia caldo. Ma Fabrizi la tratta burberamente; Maddalena quasi sviene dal dolore, ma poi Fabrizi accarezza la bambina e questo rianima Maddalena. Chi se non sua figlia sarà la prescelta?28

Nel 1950 Fabrizi è il nuovo divo, passato attraverso la rigenerazione poetica di Rossellini, che incute soggezione e riscuote elogi: i suoi modi burberi hanno il potere di gettare Maddalena in un profondo sconforto. Guitti e filodrammatici sono stati spazzati via dalla guerra, ma anche le vecchie stelle del cinema hanno lasciato il posto ai protagonisti della stagione neorealista: Rossellini, Bergman, Mangano e Fabrizi hanno preso il posto di Clara Calamai e Doris Duranti.

La scaletta di Bellissima, ultimo documento del fascicolo, può essere considerata successiva agli otto soggetti per un particolare: il personaggio di Maddalena ha ormai il cognome che porterà nel film, Cecconi, mai apparso nelle versioni precedenti ${ }^{29}$. Vengono introdotte nuove varianti: il film per cui si cerca una bambina avrà per protagonista Amedeo Nazzari, Yvonne Sanson vi reciterà il ruolo della sorella o di sua figlia, il regista sarà

del teatro, mentre guarda con gli occhi lucidi la scena che la Mangano sta girando, non protesta quando Annovazzi le mette una mano sulla spalla." (R9h 7 e Pio Baldelli 107). Sul film Riso amaro si veda anche O'Rawe (127-142).

26 “8 agosto 1949, ore 12 - Combinato con D'Angelo soggetto e sceneggiatura Prima comunione per cinque milioni”. (Pio Baldelli 162).

27 Cfr. Stephen Gundle (759-86).

28 R9h 7 e Pio Baldelli 107.

29 Il documento non ha contrassegno numerico, è semplicemente citato come "Scaletta." Si tratta di dieci pagine con note manoscritte divise in trentotto punti, corrispondenti ad altrettante descrizioni di macrosequenze narrative. Notiamo per inciso che i caratteri della macchina da scrivere non corrispondono a quelli dei documenti precedenti. 
Mario Camerini. Al punto 27 del testo compare, nel vuoto domenicale di Cinecittà, mentre Roma è assediata dal derby Roma-Lazio (situazione che rievoca il finale di Ladri di biciclette), De Sica nel ruolo di se stesso: in procinto di iniziare le riprese di un suo film, riconosce in Maddalena "una faccia scavata piena di significato" ("Scaletta" 9).

"Le piacerebbe fare una parte nel mio film?" — Maddalena lo guarda un momento, poi non resiste più e scarica i suoi nervi mollando un sonoro ceffone al regista. Poi scappa via trascinandosi dietro la figlia. De Sica rimane, colpito, a considerare la reazione della donna. Conclude col dare ragione a quella donna: "Chissà cosa aveva dentro di sé in quel momento?... E uno entra, di prepotenza, nei loro dolori, magari..."30

Lo sfondo è dunque di nuovo mutato: lo scenario neorealista si è eclissato lasciando il posto al cinema popolare dei melodrammi di Raffaele Matarazzo che nel 1950 aveva firmato il suo primo grande successo anche se, a dirigere la coppia dei divi di Catene, la scaletta vede impegnato Mario Camerini ${ }^{31}$.

Nelle varie stesure i riferimenti al mondo del cinema vengono continuamente aggiornati per fare in modo che il sogno ad occhi aperti di Maddalena acquisti un suo spessore realistico, ma le tracce di questi personaggi che recitano nel ruolo di se stessi, stratificate e sovrapposte negli anni, disegnano anche una precisa mappa autobiografica: sono tutti protagonisti del cinema italiano con cui Zavattini aveva lavorato, personaggi, come amava dire, "nome e cognome", presenze e ricordi dello scrittore legato a doppio filo all'ambiente del cinema romano da lui frequentato instancabilmente per decenni a partire, come De Sica, dagli anni Trenta: lo stesso microcosmo ad alto tasso simbolico che il suo soggetto va a stigmatizzare. La società dello spettacolo descritta da Guy Debord è appena agli albori, la televisione non ha ancora fatto il suo ingresso nelle case degli italiani (anche nel film è la radio che trasmette il bando di concorso lanciato dalla casa di produzione) eppure Zavattini, nel suo florilegio di soggetti sull'uomo comune traviato dal mondo del cinema al punto da fare del figlio uno strumento di compensazione delle proprie frustrazioni, continua a disegnare la cartografia ideale di quell'universo fatuo che affascina e soggioga le masse.

\section{0 "Scaletta" 9.}

31 Camerini, il regista dei maggiori successi attoriali di De Sica, era stato il primo per cui Zavattini aveva scritto una sceneggiatura, quella di Darò un milione (1935). 
C'è dunque una forte contraddizione nel rapporto che, attraverso i vari soggetti di Bellissima, lo scrittore luzzarese intesse con il cinema italiano. Mentre punta il dito contro l'ingenuità popolare, i suoi miti e le false speranze riposte nella fabbrica dell'immaginario, ne è avvinto e tenta di volta in volta di cooptarne gli esponenti di spicco, per lui figure quotidiane e familiari di amici e colleghi. Da alfiere della modernità cinematografica qual è, Zavattini tematizza, all'interno del suo soggetto in divenire, la macchina-cinema. Scruta dentro il suo meccanismo, oscuro allo spettatore del periodo classico, svelandone il funzionamento: fra una passerella di divi e un teatro di posa affollato di aspiranti attori fa recitare dentro il film registi e protagonisti del cinema italiano mostrandoli nel ruolo di se stessi; segue una prassi che si consoliderà più tardi, a partire dagli anni Sessanta. Nella "Scaletta" i personaggi principali finiscono preda di questa sorta di gioco di ruoli collettivo: la Spernanzoni fa la comparsa, Annovazzi viene ingaggiato come trovarobe, Maddalena riceve la proposta di De Sica, Camerini vorrebbe scritturare Maria. Arriva un momento in cui la finzione pare fagocitare la realtà, il condominio che imprigiona madre e figlia resta sempre più distante, quasi irreale - e invece più reale del vero appare il circo vivente della macchina produttiva dipinto come un Moloch insaziabile. Sul finale il can-can cessa di colpo. Madre e figlia fanno ritorno a casa, Maddalena torna all'ordine familiare scegliendo per sé e per Maria i ruoli di madre assennata e di figlia ubbidiente, rinunciando ai sogni e alle favole di celluloide: si arrende alla residenza coatta nel piccolo appartamento anonimo di periferia. Scrive Zavattini nelle ultime righe della "Scaletta": "Spartaco la guarda a lungo: non ha capito molto. Ma sente che in quel momento sua moglie ha bisogno della sua protezione, del suo aiuto. Sente che tutto è tornato in ordine." Questa conclusione, che il film di Visconti adotterà e sposerà come epilogo dell'avventura di Maddalena $\mathrm{e}$ Maria a Cinecittà, arriva improvvisa e incongrua a livello drammaturgico ma del tutto in linea con l'ideologia che domina la società italiana degli anni Cinquanta ${ }^{32}$. Il mondo di Maddalena e della piccola Maria resta cinto d'assedio dalla società dello spettacolo che preme, vuole entrare nelle vite comuni, illuderle proponendo loro una via di fuga, soggiogare e corrompere. Questa evidenza continuerà a minare la serenità familiare degli italiani per molti decenni a venire costituendo un filo sottile e resistente in grado di saldare teatro, cinema e televisione in un'unica aberrante distorsione della percezione dell'essere e dell'apparire fino a precipitare nell'incubo delineato da Reality di Matteo Garrone (2012).

32 Cfr. Giovanna Grignaffini e Piera Detassis. 
Anche se lo sfondo, secondo la logica neorealista, riveste nei soggetti di Bellissima la stessa importanza delle figure che lo abitano, la comparazione dei diversi testi ha mostrato che fino dalla prima stesura Enrico/Enrichetto e Mariù/Maria, cioè sia la versione maschile che quella femminile del personaggio infantile co-protagonista del racconto, appaiono inadeguati a rispondere alle aspettative che il genitore ha nei loro confronti. Né Enrichetto né Maria sono realmente in grado di recitare in un film. Ciò nonostante giungono a un passo dal riuscirci, ma solo per l'ossessione che muove l'adulto. I bambini appaiono privi di iniziativa perché timidi, le loro azioni vengono inibite o guidate dalla volontà dei grandi. Sono capaci soltanto di guardare, con grandi occhi timidi e ingenui, la follia che si impadronisce della persona che dovrebbe sostenerli ed educarli. È lo stesso sguardo che Bruno/Enzo Stajola esibisce in molte inquadrature di Ladri di biciclette, ma a Enrichetto e a Maria, a differenza di Bruno, viene inibita qualsiasi possibilità di azione.

Certo, Bellissima si chiude con l'inquadratura di Maria che dorme serena rischiarata dalla luce della camera dove Maddalena e Spartaco si sono appena rappacificati; d'altronde questa immagine resta portatrice di un senso ottuso evidentissimo ${ }^{33}$. Possiamo assegnarle qualsiasi valore e il risultato non cambia questa bambina resta una piccola perseguitata. La catena dei soggetti zavattiniani evidenzia che solo al momento in cui Enrichetto si trasforma in Maria il personaggio diventa pienamente succube: "subisce la mania materna" sono le parole dell'autore. Nell'ultimo testo contenuto nel fascicolo, quello di undici pagine, tale tratto distintivo del personaggio infantile diventa ancor più esplicito: "Quando giunge il suo turno, Maria entra nella stanza del regista come una vittima: perché è stanca delle lunghe ore passate lì in attesa, è stanca della levataccia cui l'ha costretta la madre per giungere tra le prime a Cinecittà; è stanca degli infiniti consigli con i quali la madre l'assilla dall'alba" (R9h 3). E la "Scaletta" rincara la dose: "Dal parrucchiere la bambina si è addormentata mentre le fanno la permanente. È tormentata da un incubo e quando si sveglia si trova davanti la maestra. In preda ad un pianto nervoso Maria chiede della mamma" ("Scaletta" 6). L'atteggiamento maniacale di Maddalena distrugge Maria e la costringe perfino a mentire: "Maria deve dire le bugie quando il padre la interroga sull'andamento della scuola" ("Scaletta" 6). Più si inoltra nell'ossessione che ormai si è impadronita della donna, più Zavattini mette l'accento sulla crudeltà da lei esercitata sulla bambina: "Ha portato Maria dai migliori fotografi di Roma e la piccola era così stanca che davanti alla mac-

33 Il riferimento è a Roland Barthes. 
china fotografica non riusciva neanche più a sorridere" ("Scaletta" 9). Non solo, si provvede a precisare che le altre madri sparse per i corridoi di Cinecittà si stanno comportando esattamente come Maddalena. Solo quando la prova di Maria risulta "fra le peggiori" e il regista Blasetti e il maneggione Annovazzi pronunciano giudizi offensivi su sua figlia, Maddalena riesce a cacciare via il demone che si è impadronito di lei e "capisce anche tante altre cose: che solo per ambizione ha fatto una serie di così grosse sciocchezze, ha torturato, si può dire, sua figlia" ("Scaletta"11. Il corsivo è mio). In tutto questo, Maria "le trotterella vicino silenziosa" (Ibidem). L'unica trasgressione che le è concessa, una volta salita sul tram che le riconduce a casa, consiste nell'affacciarsi fuori dal finestrino, vicino alla madre il cui volto è solcato da un paio di lacrime, giusto perché la mamma l'ha invitata a farlo: da sola non avrebbe osato sporgersi, specifica Zavattini.

Nella storia di Maddalena e Maria, così come delineata nell'ultimo segmento della lunga scia testuale, emerge la profonda trasformazione dei piccoli protagonisti dei film neorealisti maturata nel passaggio dal periodo postbellico alla svolta politica e culturale avvenuta in Italia alla fine degli anni Quaranta. Se la novità de I bambini ci guardano consisteva nel consegnare a Pricò lo sguardo, ingenuo ma limpido, sull'universo familiare in rovina, compreso un severo giudizio sull'operato della madre, l'ultimo soggetto di Bellissima racconta l'impotenza di Maria, "torturata" da Maddalena e ciò nonostante vittima passiva e inconsapevole, incapace non solo di ribellarsi ma neanche di accorgersi del sopruso che patisce. Questo "ciocco di carne" di pirandelliana memoria appartiene a un altro mondo, a un'altra epoca, delinea uno scenario alternativo rispetto a quello rappresentato dalla straordinaria vitalità e iniziativa dei bambini cinematografici scaturiti dalla fantasia dello Zavattini neorealista. La placida passività di Maria e la contrizione finale di Maddalena raccontano il luogo perfetto, nell'Italia dominata dalla Democrazia Cristiana, della restaurazione ideologica che passa attraverso il monito severo dell'obbedienza e la rinuncia alle tentazioni in special modo per le donne.

\section{Università di Firenze}

\section{Opere citate}

Alovisio, Silvio. "La spettatrice muta. Il pubblico cinematografico femminile nell'Italia del primo Novecento" Non solo dive. Pioniere del cinema italiano. A cura di Monica Dall'Asta. Bologna: Cineteca di Bologna, 2008. 269-286.

Baldelli, Pio. Luchino Visconti. Milano: Mazzotta, 1973.

Barthes, Roland. L'obvie et l'obtus. Essais critiques III. Paris: Éditions du Seuil, 1982. 
Bernardi, Sandro. "L'attenzione al cinema nelle riviste di critica teatrale: Comoedia, Scenario e altre ancora" AA.VV., Nuovi materiali sul cinema italiano 1929-43. vol. I. Quaderno Informativo Mostra Internazionale del Nuovo Cinema di Pesaro. Venezia: Marsilio, 1976. 79-87.

Bernardini, Aldo, Gili, Jean. Cesare Zavattini. Parigi: Centre Georges Pompidou, 1990.

Borin, Fabrizio. "L'attore con la macchina da presa" Cinematecnica. Percorsi critici nella fabbrica dell'immaginario. A cura di Id., Roberto Ellero. Roma: Bulzoni, 2001. 269-90

Bravo, Anna. Donne e uomini nelle guerre mondiali. Roma-Bari: Laterza, 1995.

Bruzzone, Anna Maria. In guerra senz'armi. Storie di donne. 1940-1945. RomaBari: Laterza, 1995.

Caldiron, Orio. Il paradosso dell'autore. Roma: Bulzoni, 1999.

Cardone, Lucia. "Madri" Quaderni del CSCI 9 (2013): 204-207. Debord, Guy. La société due spectacle. Paris: Buchet-Chastel, 1967.

Detassis, Piera "Corpi recuperati per il proprio sguardo. Cinema e immaginario negli anni Cinquanta” Memoria, 6 (1982) : 24-31.

De Vincenti, Giorgio. Il concetto di modernità nel cinema. Parma: Pratiche, 1993. Ferrone, Siro. "Bellissima: l'educazione teatrale" Drammaturgia 7 (2000): 83-95. Grande, Maurizio, "Il soggetto inesauribile" Cesare Zavattini, La veritàaaa. Milano: Bompiani,1983. 7-65.

Grignaffini, Giovanna "Female identity and Italian Cinema of the 1950s" Off Screen: Women and film in Italy. A cura di Giuliana Bruno, Maria Nadotti, Laura Mulvey. London: Routledge, 1988. 111-123.

Gundle, Stephen "Il divismo nel cinema europeo, 1945-1960" Storia del cinema mondiale. A cura di Gian Piero Brunetta, vol. 1. Torino: Einaudi, 1999. 759-86. Jandelli, Cristina. La scena pensante. Cesare Zavattini fra teatro e cinema. Roma: Bulzoni, 2002.

Marcus, Millicent. Italian Film in the Light of Neorealism. Princeton: Princeton University Press, 1986.

Mazzei, Luca. "Al cinematografo da sole. Il cinema descritto dalle donne fra 1898 e 1916" Non solo dive. Pioniere del cinema italiano. A cura di Monica Dall'Asta. Bologna: Cineteca di Bologna, 2008. 257-268.

Micciché, Lino Visconti e il neorealismo. Ossessione, La terra trema, Bellissima. Venezia: Marsilio, 1998.

Muraro, Luisa. L'ordine simbolico della madre. Roma: Editori Riuniti, 1992.

Nuzzi, Paolo, Ottavio Iemma. De Sica \& Zavattini. Parliamo tanto di noi. Roma: Editori Riuniti, 1997.

O'Rawe, Catherine "Gender, Genre and Stardom: Fatality in Italian Neorealist Cinema" The Femme Fatale: Images, Histories, Context. A cura di Helen Hanson, Catherine O’Rawe. London: Palgrave Macmillan, 2010.

Parigi, Stefania. Fisiologia dell'immagine. Il pensiero di Cesare Zavattini. Torino: Lindau, 2008.

Vicentini, Claudio. L'arte di guardare gli attori. Venezia: Marsilio, 2007.

Zavattini, Cesare "Aldo Fabrizi” Tempo, II, 42 (1940): 39 
—_, "Inaugurazione del Reale" Tempo, II, 78 (1940): 41

Io. Un'autobiografia. A cura di Paolo Nuzzi. Torino: Einaudi, 2002.

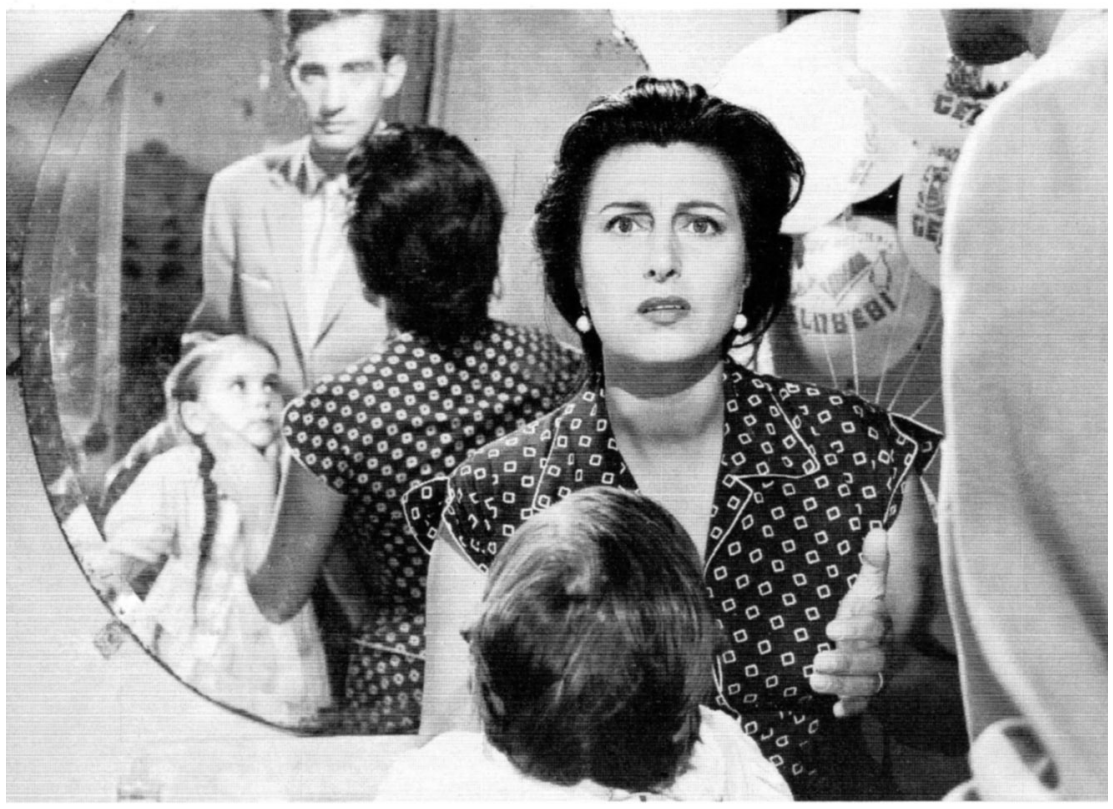

Anna Magnani in un'immagine di Bellissima (1951) di Luchino Visconti da un soggetto di Cesare Zavattini. 